\title{
Reliable Control for Uncertain Singular Systems with Randomly Occurring Time-Varying Delay and Actuator Faults
}

\author{
Yu-Lin Li, Lin-Sheng Li, Zhi-Cheng Zhao, and Jing-Gang Zhang \\ Taiyuan University of Science and Technology, Taiyuan 030024, China \\ Correspondence should be addressed to Lin-Sheng Li; llsheng@163.com
}

Received 13 August 2014; Accepted 30 August 2014

Academic Editor: Zheng-Guang Wu

Copyright ( 2015 Yu-Lin Li et al. This is an open access article distributed under the Creative Commons Attribution License, which permits unrestricted use, distribution, and reproduction in any medium, provided the original work is properly cited.

\begin{abstract}
The problem of reliable control is investigated for uncertain continuous singular systems with randomly occurring time-varying delay and actuator faults in this work. The delay occurs in a random way, and such randomly occurring delay obeys certain mutually uncorrelated Bernoulli distributed white noise sequences. The uncertainties under consideration are norm-bounded, and may vary with time. Then, with the constructed Lyapunov function, a sufficient condition is given to ensure the unforced system is meansquare exponentially stable and the corresponding controller can be derived from such condition, and the actuator faults problem is guaranteed. A numerical example is provided to show the effectiveness of the methods.
\end{abstract}

\section{Introduction}

During the past three decades, the studies of singular systems have been an active field of research in many scientific and technical disciplines. Dynamic input-output model, electronic network, constrained robots, nuclear reactors, and other noncausal systems all belong to the typical singular systems. Recently, the problems of robust stability analysis and robust stabilization for singular systems have been studied. It is worth noticing that the robust stability problem for singular systems is much more complicated than that for regular systems because it requires considering not only stability robustness, but also regularity and absence of impulses (for continuous singular systems) and causality (for discretesingular systems) at the same time, and the latter two need not be considered in regular systems. There are lots of papers that have studied these subjects [1-3].

On the other hand, singular systems with time-delays arise in a variety of practical systems such as chemical processes and lossless transmission lines. Since singular systems with time-delays are matrix delay differential equations coupled with matrix difference equations, the study of such systems is much more complicated than that of standard state-space time-delay systems or singular systems. The existence and uniqueness of a solution to a given singular time-delay system is not always guaranteed. In accordance with the advance of robust control theory, a number of robust stabilization methods have been proposed for uncertain timedelay systems [4-9].

For continuous singular systems, a few of the studies have mentioned the robust stabilization of the uncertain timedelay system. In this paper, we address the problems of robust stability and stabilization for uncertain singular systems with randomly occurring time-varying delay (ROTD). Although the randomly occurring delay has appeared in some papers [ 1 , $10,11]$, few related results have been established for uncertain singular systems.

Recently, much effort has been devoted to the reliable control with unexpected failures which were often found in the real world. Therefore, designing a controller which could tolerate some actuator failures has been investigated for dynamical systems, discrete-time fuzzy system, networked control system, and so forth. Up to now, the issue of reliable control for uncertain singular systems with randomly occurring time-varying delay and actuator failures has not been fully investigated.

In this paper, we deal with the problem of reliable control and exponential stability analysis for uncertain singular systems with ROTD and actuator faults. A random variable, which obeys Bernoulli distribution, is introduced to 
account for ROTD. By the LMI approach, a state feedback controller is established to guarantee the resultant closedloop system is delay-dependent exponentially admissible. Finally, a numerical example is given to show the usefulness of the result derived.

Notations. $R^{n}$ denotes the $n$-dimensional Euclidean space and $R^{m \times n}$ is the set of all $m \times n$ real matrices. The notation $X>Y(X \geq Y)$, where $X$ and $Y$ are symmetric matrices, means that $X-Y$ is positive definite (positive semidefinite). $I$ and 0 represent the identity matrix and a zero matrix, respectively; and $\operatorname{diag}\{\cdots\}$ stands for a block-diagonal matrix. The superscript " $T$ " represents the transpose and the asterisk "*” in a matrix is used to represent the term which is induced by symmetry. $\mathbb{E}\{x\}$ means the expectation of the stochastic variable $x$.

\section{Problem Formulation and Preliminaries}

Consider the uncertain singular system with randomly occurring time-varying delay:

$$
\begin{aligned}
E \dot{x}(t)= & (A+\Delta A) x(t) \\
& +\alpha(t)\left(A_{d}+\Delta A_{d}\right) x(t-d(t)) \\
& +(B+\Delta B) u^{F}(t) \\
x(t)= & \phi(t), \quad t \in[-d, 0],
\end{aligned}
$$

where $x(t) \in R^{n}$ is the state vector and $u(t) \in R^{m}$ is the control input vector. $\phi(t)$ is a compatible vector valued initial function. The matrix $E \in R^{n \times n}$ may be singular and it is assumed that $\operatorname{rank}(E)=r \leq n . d(t)$ is a time-varying continuous function that satisfies $0<d(t) \leq d$ and $\dot{d}(t) \leq \mu$, where 0 and $d$ are the lower and upper bounds of the time-delay $d(t) . A, A_{d}$, and $B$ are known real constant matrices with appropriate dimensions. $\Delta A, \Delta A_{d}$, and $\Delta B$ are unknown matrices representing norm-bounded parametric uncertainties and are assumed to be of the form

$$
\left[\begin{array}{lll}
\Delta A & \Delta A_{d} & \Delta B
\end{array}\right]=\operatorname{MF}(t)\left[\begin{array}{lll}
N_{1} & N_{2} & N_{3}
\end{array}\right]
$$

where $M, N_{1}, N_{2}$, and $N_{3}$ are known real constant matrices with appropriate dimensions and $F(t) \in R^{q \times k}$ is an unknown real and possibly time-varying matrix satisfying

$$
F(t)^{T} F(t) \leq I \text {. }
$$

The parametric uncertainties $\Delta A, \Delta A_{d}$, and $\Delta B$ are said to be admissible if both (2) and (3) hold.

To account for the phenomena of ROTD, we introduce the stochastic variable $\alpha(t)$, which is a Bernoulli-distributed white sequence. The natural assumption on $\alpha(t)$ is as follows:

$$
\begin{aligned}
& \operatorname{Prob}\{\alpha(t)=1\}=\mathbb{E}\{\alpha(t)\}=\alpha, \\
& \operatorname{Prob}\{\alpha(t)=0\}=1-\mathbb{E}\{\alpha(t)\}=1-\alpha,
\end{aligned}
$$

where the constant $\alpha \in[0,1]$.
When the actuators experience failures, we use $u^{F}(t)$ to describe the control signal sent from actuators. Consider the actuator failure model with failure parameter

$$
u^{F}(t)=W u(t)
$$

$W=\operatorname{diag}\left\{w_{1}, w_{2}, \ldots, w_{m}\right\}$ is the actuator failure matrix with the following property:

$$
0 \leq w_{i}^{\min } \leq w_{i} \leq w_{i}^{\max } \leq 1, \quad i=1,2, \ldots, m,
$$

where

$$
\begin{array}{r}
w_{i}^{\min }=w_{i}^{\max }=1, \quad i=1,2, \ldots, m \\
\Longrightarrow i \text { th actuator has no failure }
\end{array}
$$

$$
w_{i}^{\min }=w_{i}^{\max }=0, \quad i=1,2, \ldots, m
$$

$\Longrightarrow i$ th actuator is outage

$$
\begin{aligned}
0< & w_{i}<1, \quad i=1,2, \ldots, m \\
& \Longrightarrow i \text { th actuator has partial failure. }
\end{aligned}
$$

Define the following notations:

$$
\begin{aligned}
W_{0} & =\operatorname{diag}\left\{w_{01}, w_{02}, \ldots, w_{0 m}\right\}, \\
H & =\operatorname{diag}\left\{h_{1}, h_{2}, \ldots, h_{m}\right\}, \\
G & =\operatorname{diag}\left\{g_{1}, g_{2}, \ldots, g_{m}\right\}, \\
|G| & =\operatorname{diag}\left\{\left|g_{1}\right|,\left|g_{2}\right|, \ldots,\left|g_{m}\right|\right\},
\end{aligned}
$$

where

$$
\begin{gathered}
w_{0 i}=\frac{w_{i}^{\max }+w_{i}^{\min }}{2}, \\
h_{i}=\frac{w_{i}^{\max }-w_{i}^{\min }}{w_{i}^{\max }+w_{i}^{\min }} \\
g_{i}=\frac{w_{i}-w_{0 i}}{w_{0 i}}, \\
i=1,2, \ldots, m .
\end{gathered}
$$

From notations $(8 \mathrm{a})-(8 \mathrm{~b})$, we have

$$
W=W_{0}(I+G), \quad|G| \leq H \leq I .
$$

Because actuator failure that might occur is unknown, the matrix $M$ is not well known in advance. The matrix $M$ satisfies (6) in which the matrix $M_{0}$ is known in advance.

The nominal unforced singular systems with randomly occurring time-varying delay of (1) can be described as follows:

$$
\begin{aligned}
E \dot{x}(t) & =A x(t)+\alpha(t) A_{d} x(t-d(t)), \\
x(t) & =\phi(t), \quad t \in[-d, 0] .
\end{aligned}
$$

Definition 1. System (11) is said to be regular and impulsefree, if the pair $(E, A)$ is regular and impulse-free. 
Definition 2. System (11) is said to be exponentially stable, if there exist scalars $\alpha>0$ and $\beta>0$ such that $\|x(t)\| \leq$ $\alpha e^{-\beta t}\|\phi(t)\|_{d}$.

Definition 3. System (11) is said to be exponentially admissible, if it is regular, impulse-free, and exponentially stable.

Definition 4. The uncertain singular system (1) is said to be exponentially admissible, if the unforced system is regular, impulse-free, and exponentially stable for all admissible uncertainties $\Delta A$ and $\Delta A_{d}$.

Let us consider the following controller:

$$
u(t)=K x(t)
$$

where $K \in R^{m \times n}$ is the controller gain matrix to be designed.

Substituting $u^{F}(t)$ for $u(t)$ in (1) and considering (5) and (12), the closed-loop system can be described by

$$
\begin{aligned}
E \dot{x}(t)= & (A+B W K+\Delta A+\Delta B W K) x(t) \\
& +\alpha(t)\left(A_{d}+\Delta A_{d}\right) x(t-d(t)), \\
x(t)= & \phi(t), \quad t \in[-d, 0] .
\end{aligned}
$$

Lemma 5 (Lu et al. [12]). For any matrix $M>0$, integers $\gamma_{1}$ and $\gamma_{2}$ satisfying $\gamma_{2}>\gamma_{1}$, and vector function $\omega: N\left[\gamma_{1}, \gamma_{2}\right] \rightarrow$ $R^{n}$, such that the sums concerned are well defined,

$$
\begin{aligned}
& \left(\gamma_{2}-\gamma_{1}\right) \int_{\gamma_{1}}^{\gamma_{2}} \omega(\alpha)^{T} M \omega(\alpha) d \alpha \\
& \geq\left[\int_{\gamma_{1}}^{\gamma_{2}} \omega(\alpha) d \alpha\right]^{T} M\left[\int_{\gamma_{1}}^{\gamma_{2}} \omega(\alpha) d \alpha\right] .
\end{aligned}
$$

Lemma 6 (Wu and Zheng [13]). Given matrices $\Xi, \Gamma$, and $\Phi$ with appropriate dimensions and with $\Xi$ symmetrical, then $\Xi+$ $H F \Phi+\Phi^{T} F^{T} H^{T}<0$ for any $F$ satisfying $F^{T} F \leq I$, if and only if there exists a scalar $\varepsilon>0$ such that $\Xi+\varepsilon H H^{T}+\varepsilon^{-1} \Phi^{T} \Phi<0$.

Lemma 7 (Mao et al. [9]). Suppose that a positive continuous function $f(t)$ satisfies

$$
f(t) \leq \zeta_{1} \sup _{t-d \leq s \leq t} f(t)+\zeta_{2} e^{-\varepsilon t}
$$

where $\varepsilon>0,0<\zeta_{1}<1,0<\zeta_{1} e^{\varepsilon d}<1, \zeta_{2}>0$, and $d>0$; then,

$$
f(t) \leq e^{-\varepsilon t}\|f(s)\|_{d}+\frac{\zeta_{2} e^{-\varepsilon t}}{1-\zeta_{1} e^{\varepsilon d}}
$$

\section{Main Result}

In this section, we first derive a condition to guarantee nominal unforced system (11) to be mean-square exponential stability.
Theorem 8. Singular time-delay system (11) is exponentially robustly stable, if there exist matrices $P, Y, Q>0, R>0$, and $Z>0$ such that

$$
\begin{aligned}
E^{T} P & =P^{T} E \geq 0, \\
\Xi & =\left[\begin{array}{cccc}
\Xi_{11} & \Xi_{12} & E^{T} Y E & d A^{T} Z \\
* & \Xi_{22} & -E^{T} Y E+E^{T} Z E & d \alpha A_{d}^{T} Z \\
* & * & -R & 0 \\
* & * & * & -Z
\end{array}\right] \\
& <0,
\end{aligned}
$$

$$
\left[\begin{array}{ll}
Z & Y \\
* & Z
\end{array}\right]>0
$$

where

$$
\begin{aligned}
& \Xi_{11}=P^{T} A+A^{T} P+Q+R-E^{T} Z E, \\
& \Xi_{12}=\alpha P^{T} A_{d}+E^{T} Z E-E^{T} Y E, \\
& \Xi_{22}=-(1-\mu) Q+E^{T} Y E+E^{T} Y^{T} E-2 E^{T} Z E .
\end{aligned}
$$

Proof. Firstly, the regularity and absence of impulses of system (8a), (8b), (8c), and (8d) are given. For singular system, we know that there exist nonsingular matrices $G$ and $H$ such that

$$
G E H=\left[\begin{array}{cc}
I_{r} & 0 \\
0 & 0
\end{array}\right]
$$

Denote

$$
\begin{aligned}
G A H & =\left[\begin{array}{ll}
A_{1} & A_{2} \\
A_{3} & A_{4}
\end{array}\right], \\
G^{-T} P H & =\left[\begin{array}{ll}
P_{1} & P_{2} \\
P_{3} & P_{4}
\end{array}\right] .
\end{aligned}
$$

From (17a) and using the expressions in (19) and (20), it can be found that $P_{2}=0$. Then, premultiplying and postmultiplying $\Xi_{11}<0$ by $H^{T}$ and $H$, respectively, we have

$$
A_{4}^{T} P_{4}+P_{4}^{T} A_{4}<0
$$

which implies $A_{4}$ is nonsingular and thus the pair $(E, A)$ is regular and impulse-free. Hence, by Definition 1, system (11) is regular and impulse-free.

Then, the problem for the unforced system (11) to be exponentially stable is given. Consider the following Lyapunov function for system (11):

$$
V\left(x_{t}\right)=V_{1}\left(x_{t}\right)+V_{2}\left(x_{t}\right)+V_{3}\left(x_{t}\right),
$$

where

$$
\begin{aligned}
V_{1}\left(x_{t}\right)= & x(t)^{T} E^{T} P x(t), \\
V_{2}\left(x_{t}\right)= & \int_{t-d(t)}^{t} x(s)^{T} \mathrm{Q} x(s) d s \\
& +\int_{t-d}^{t} x(s)^{T} R x(s) d s, \\
V_{3}\left(x_{t}\right)= & d \int_{-d}^{0} \int_{t+\theta}^{t} \dot{x}(s) E^{T} Z E \dot{x}(s) d s d \theta .
\end{aligned}
$$


Define the infinitesimal operator $\mathbb{L}$ of $V\left(x_{t}\right)$ as follows:

$$
\mathbb{L} V\left(x_{t}\right)=\lim _{h \rightarrow 0^{+}} \frac{1}{h}\left\{\mathbb{E}\left\{V\left(x_{t+h}\right) \mid x_{t}\right\}-V\left(x_{t}\right)\right\} .
$$

We obtain

$$
\begin{aligned}
\mathbb{E}\left\{\mathbb{L} V\left(x_{t}\right)\right\}= & \mathbb{E}\left\{\mathbb{L} V_{1}\left(x_{t}\right)\right\}+\mathbb{E}\left\{\mathbb{L} V_{2}\left(x_{t}\right)\right\} \\
& +\mathbb{E}\left\{\mathbb{L} V_{3}\left(x_{t}\right)\right\},
\end{aligned}
$$

where

$$
\begin{aligned}
& \mathbb{E}\left\{\mathbb{L} V_{1}\left(x_{t}\right)\right\} \\
& =\mathbb{E}\left\{2 x(t)^{T} E^{T} P \dot{x}(t)\right\} \\
& =\mathbb{E}\left\{2 x(t)^{T} E^{T} P\left(A x(t)+\alpha(t) A_{d} x(t-d(t))\right)\right\} \\
& =2 x(t)^{T} E^{T} P\left(A x(t)+\alpha A_{d} x(t-d(t))\right) \\
& \mathbb{E}\left\{\mathbb{L} V_{2}\left(x_{t}\right)\right\} \\
& \leq \mathbb{E}\left\{x(t)^{T} Q x(t)-(1-\mu) x(t-d(t))^{T}\right. \\
& \text { - } \left.Q x(t-d(t))+x(t)^{T} R x(t)-x(t-d)^{T} R x(t-d)\right\} \\
& =x(t)^{T} \mathrm{Q} x(t)-(1-\mu) x(t-d(t))^{T} \mathrm{Q} x(t-d(t)) \\
& +x(t)^{T} R x(t)-x(t-d)^{T} R x(t-d) \\
& \mathbb{E}\left\{\mathbb{L} V_{3}\left(x_{t}\right)\right\} \\
& =\mathbb{E}\left\{d^{2} \dot{x}(t)^{T} E^{T} Z E \dot{x}(t)-d \int_{t-d}^{t} \dot{x}(t)^{T} E^{T} Z E \dot{x}(t) d s\right\} \\
& =d^{2}\left(A x(t)+\alpha A_{d} x(t-d(t))\right)^{T} \\
& \cdot Z\left(A x(t)+\alpha A_{d} x(t-d(t))\right) \\
& -d \int_{t-d}^{t} \dot{x}(t)^{T} E^{T} Z E \dot{x}(t) d s
\end{aligned}
$$

Applying Lemma 5, when $0<d(t)<d$, we have that

$$
\begin{aligned}
& -d \int_{t-d}^{t} \dot{x}(t)^{T} E^{T} Z E \dot{x}(t) d s \\
& =-d \int_{t-d(t)}^{t} \dot{x}(t)^{T} E^{T} Z E \dot{x}(t) d s \\
& -d \int_{t-d}^{t-d(t)} \dot{x}(t)^{T} E^{T} Z E \dot{x}(t) d s
\end{aligned}
$$

$$
\leq-\frac{d}{d(t)} \int_{t-d(t)}^{t} \dot{x}(t)^{T} E^{T} d s Z \int_{t-d(t)}^{t} E \dot{x}(t) d s
$$$$
-\frac{d}{d-d(t)} \int_{t-d}^{t-d(t)} \dot{x}(t)^{T} E^{T} d s Z \int_{t-d}^{t-d(t)} E \dot{x}(t) d s
$$$$
=-\int_{t-d(t)}^{t} \dot{x}(t)^{T} E^{T} d s Z \int_{t-d(t)}^{t} E \dot{x}(t) d s
$$$$
-\int_{t-d}^{t-d(t)} \dot{x}(t)^{T} E^{T} d s Z \int_{t-d}^{t-d(t)} E \dot{x}(t) d s
$$$$
-\frac{d-d(t)}{d(t)} \int_{t-d(t)}^{t} \dot{x}(t)^{T} E^{T} d s Z \int_{t-d(t)}^{t} E \dot{x}(t) d s
$$$$
-\frac{d-d(t)}{d(t)} \int_{t-d(t)}^{t} \dot{x}(t)^{T} E^{T} d s Z \int_{t-d(t)}^{t} E \dot{x}(t) d s
$$$$
-\frac{d-d(t)}{d(t)} \int_{t-d(t)}^{t} \dot{x}(t)^{T} E^{T} d s Z \int_{t-d(t)}^{t} E \dot{x}(t) d s
$$$$
-\frac{d(t)}{d-d(t)} \int_{t-d}^{t-d(t)} \dot{x}(t)^{T} E^{T} d s Z \int_{t-d}^{t-d(t)} E \dot{x}(t) d s .
$$

It can be from (17c) that (29)

$$
\begin{aligned}
& {\left[\begin{array}{l}
\sqrt{\frac{d-d(t)}{d(t)}} \int_{t-d(t)}^{t} E \dot{x}(t) d s \\
-\sqrt{\frac{d(t)}{d-d(t)}} \int_{t-d}^{t-d(t)} E \dot{x}(t) d s
\end{array}\right]^{T}\left[\begin{array}{ll}
Z & Y \\
* & Z
\end{array}\right]} \\
& {\left[\begin{array}{c}
\sqrt{\frac{d-d(t)}{d(t)}} \int_{t-d(t)}^{t} E \dot{x}(t) d s \\
-\sqrt{\frac{d(t)}{d-d(t)}} \int_{t-d}^{t-d(t)} E \dot{x}(t) d s
\end{array}\right]>0}
\end{aligned}
$$

which implies

$$
\begin{aligned}
& -\frac{d-d(t)}{d(t)} \int_{t-d(t)}^{t} \dot{x}(t)^{T} E^{T} d s Z \int_{t-d(t)}^{t} E \dot{x}(t) d s \\
& -\frac{d(t)}{d-d(t)} \int_{t-d}^{t-d(t)} \dot{x}(t)^{T} E^{T} d s Z \int_{t-d}^{t-d(t)} E \dot{x}(t) d s \\
& \leq-\int_{t-d(t)}^{t} \dot{x}(t)^{T} E^{T} d s Y \int_{t-d}^{t-d(t)} E \dot{x}(t) d s \\
& -\int_{t-d}^{t-d(t)} \dot{x}(t)^{T} E^{T} d s Y^{T} \int_{t-d(t)}^{t} E \dot{x}(t) d s .
\end{aligned}
$$


We can get from (27) and (29) that

$$
\begin{aligned}
& -d \int_{t-d}^{t} \dot{x}(t)^{T} E^{T} Z E \dot{x}(t) d s \\
& \leq-\left[\begin{array}{l}
\int_{t-d(t)}^{t} E \dot{x}(t) d s \\
\int_{t-d}^{t-d(t)} E \dot{x}(t) d s
\end{array}\right]^{T}\left[\begin{array}{ll}
Z & Y \\
* & Z
\end{array}\right]\left[\begin{array}{l}
\int_{t-d(t)}^{t} E \dot{x}(t) d s \\
\int_{t-d}^{t-d(t)} E \dot{x}(t) d s
\end{array}\right] \\
& =\left[\begin{array}{c}
x(t) \\
x(t-d(t)) \\
x(t-d)
\end{array}\right]^{T} \\
& \times\left[\begin{array}{ccc}
-E^{T} Z E & E^{T} Z E-E^{T} Y E & E^{T} Y E \\
* & -2 E^{T} Z E+E^{T} Y E+E^{T} Y^{T} E & -E^{T} Y E+E^{T} Z E \\
* & * & -E^{T} Z E
\end{array}\right] \\
& \times\left[\begin{array}{c}
x(t) \\
x(t-d(t)) \\
x(t-d)
\end{array}\right] .
\end{aligned}
$$

Then,

$$
\begin{aligned}
\mathbb{E}\{ & \left.\mathbb{L} V_{3}\left(x_{t}\right)\right\} \\
\leq & d^{2}\left(A x(t)+\alpha A_{d} x(t-d(t))\right)^{T} Z \\
& \cdot\left(A x(t)+\alpha A_{d} x(t-d(t))\right) \\
& -x(t)^{T} E^{T} Z E x(t)+2 x(t)^{T}\left(E^{T} Z E-E^{T} Y E\right) \\
& \cdot Z x(t-d(t))+2 x(t)^{T} E^{T} Y E x(t-d) \\
& +x(t-d(t))^{T}\left(-2 E^{T} Z E+E^{T} Y E+E^{T} Y^{T} E\right) x(t-d(t)) \\
& +2 x(t-d(t))^{T}\left(-E^{T} Y E+E^{T} Z E\right) x(t) \\
& -x(t-d)^{T} E^{T} Z E x(t-d)=\nabla .
\end{aligned}
$$

So

$$
\begin{aligned}
\mathbb{E}\left\{\mathbb{L} V\left(x_{t}\right)\right\}= & \mathbb{E}\left\{\mathbb{L} V_{1}\left(x_{t}\right)\right\}+\mathbb{E}\left\{\mathbb{L} V_{2}\left(x_{t}\right)\right\}+\mathbb{E}\left\{\mathbb{L} V_{3}\left(x_{t}\right)\right\} \\
\leq & 2 x(t)^{T} E^{T} P\left(A x(t)+\alpha A_{d} x(t-d(t))\right) \\
& +x(t)^{T} Q x(t) \\
& -(1-\mu) x(t-d(t))^{T} Q x(t-d(t)) \\
& +x(t)^{T} R x(t)-x(t-d)^{T} R x(t-d)+\nabla \\
= & \mathbb{E}\left\{\zeta(t)^{T} \Upsilon \zeta(t)\right\},
\end{aligned}
$$

where

$$
\begin{aligned}
\zeta(t)= & {\left[\begin{array}{c}
x(t) \\
x(t-d(t)) \\
x(t-d)
\end{array}\right], } \\
\Upsilon= & {\left[\begin{array}{ccc}
\Xi_{11} & \Xi_{12} & E^{T} Y E \\
* & \Xi_{22} & -E^{T} Y E+E^{T} Z E \\
* & * & -R
\end{array}\right] } \\
& +\left[\begin{array}{c}
A^{T} \\
A_{d}^{T} \\
0
\end{array}\right] d^{2} Z\left[\begin{array}{c}
A^{T} \\
A_{d}^{T} \\
0
\end{array}\right]^{T} .
\end{aligned}
$$

By application of Schur complement and (17b), we have $\Upsilon<$ 0 , which guarantees there exists a scalar $\lambda>0$ such that

$$
\mathbb{E}\left\{\mathbb{L} V\left(x_{t}\right)\right\}<0 .
$$

Set

$$
\widehat{G}=\left[\begin{array}{cc}
I_{r} & -A_{2} A_{4}^{-1} \\
0 & A_{4}^{-1}
\end{array}\right] G .
$$

It is easy to get

$$
\begin{aligned}
& \widehat{G} E H=\left[\begin{array}{ll}
I_{r} & 0 \\
0 & 0
\end{array}\right], \\
& \widehat{G} A H=\left[\begin{array}{ll}
\widehat{A}_{1} & 0 \\
\widehat{A}_{3} & I
\end{array}\right],
\end{aligned}
$$

where $\widehat{A}_{1}=A_{1}-A_{2} A_{4}^{-1} A_{3}$ and $\widehat{A}_{3}=A_{4}^{-1} A_{3}$.

Consider

$$
\begin{aligned}
\widehat{G} A_{d} H & =\left[\begin{array}{ll}
A_{d 1} & A_{d 2} \\
A_{d 3} & A_{d 4}
\end{array}\right], \\
\widehat{G}^{-1} P H & =\left[\begin{array}{ll}
P_{1} & P_{2} \\
P_{3} & P_{4}
\end{array}\right], \\
H^{T} \mathrm{Q} H & =\left[\begin{array}{ll}
Q_{11} & Q_{21} \\
* & Q_{22}
\end{array}\right] .
\end{aligned}
$$

It can be seen from (17b) that

$$
\left[\begin{array}{l}
I \\
I \\
I
\end{array}\right]^{T}\left[\begin{array}{ccc}
\Xi_{11} & \Xi_{12} & E^{T} Y E \\
* & \Xi_{22} & -E^{T} Y E+E^{T} Z E \\
* & * & -R
\end{array}\right]\left[\begin{array}{c}
I \\
I \\
I
\end{array}\right]<0,
$$

which implies

$$
P^{T}\left(A+\alpha A_{d}\right)+\left(A+\alpha A_{d}\right)^{T} P<0 .
$$

It is easy to find that matrix $P$ satisfying the above inequality is nonsingular. Thus, considering (17a), we can deduce that $P_{1}>0$ and $P_{2}=0$.

Define

$$
\widehat{\varsigma}(t)=\left[\begin{array}{l}
\varsigma_{1}(t) \\
\varsigma_{2}(t)
\end{array}\right]=H^{-1} x(t) .
$$


Then, system (11) is equivalent to

$$
\begin{aligned}
\dot{\zeta}(t)= & \widehat{A}_{1} \varsigma_{1}(t)+\alpha(t) A_{d 1} \varsigma_{1}(t-d(t)) \\
& +\alpha(t) A_{d 2} \varsigma_{2}(t-d(t)) \\
-\varsigma_{2}(t)= & \widehat{A}_{3} \varsigma_{1}(t)+\alpha(t) A_{d 3} \varsigma_{1}(t-d(t)) \\
& +\alpha(t) A_{d 4} \varsigma_{2}(t-d(t)) \\
\widehat{\varsigma}(t)= & \Psi(t)=H^{-1} \phi(t), \quad t \in[-d, 0] .
\end{aligned}
$$

To prove the exponential stability of system (11), we define a function as

$$
W\left(x_{t}, t\right)=e^{\varepsilon t} V\left(x_{t}, t\right),
$$

where the scalar $\varepsilon>0$

Taking its time derivative yields

$$
\begin{aligned}
\dot{W}\left(x_{t}, t\right)= & \varepsilon e^{\varepsilon t} V\left(x_{t}, t\right)+e^{\varepsilon t} \dot{V}\left(x_{t}, t\right) \leq \varepsilon e^{\varepsilon t} V\left(x_{t}, t\right) \\
& -\lambda e^{\varepsilon t}\|x(t)\|^{2}
\end{aligned}
$$

Integrating both sides of (39) from 0 to $t$, we get that

$$
\begin{aligned}
W\left(x_{t}, t\right) \leq & W\left(x_{0}, 0\right) \\
& +\int_{0}^{t} e^{\varepsilon s}\left[\varepsilon \mathbb{E}\left\{\mathbb{L} V\left(x_{s}\right)\right\}-\lambda\|x(t)\|^{2}\right] d s .
\end{aligned}
$$

By using the similar analysis method of [14], it can be seen from (22), (42), and (44) that if the scalar $\varepsilon$ is chosen small enough, a scalar $k>0$ can be found such that, for any $t>0$,

$$
V\left(x_{t}, t\right) \leq k e^{-\varepsilon t}\|\phi(t)\|_{d}^{2} .
$$

Since $\lambda_{\text {min }}\left(P_{1}\right)\left\|\varsigma_{1}(t)\right\|^{2} \leq x(t)^{T} E^{T} P x(t) \leq V\left(x_{t}, t\right)$, it can be shown from (43) that for any $t>0$

$$
\left\|\varsigma_{1}(t)\right\|^{2} \leq \beta e^{-\varepsilon t}\|\phi(t)\|_{d}^{2}
$$

where $\beta=\lambda_{\min }\left(P_{1}\right) k$. Define

$$
e(t)=\widehat{A}_{3} \varsigma_{1}(t)+\alpha(t) A_{d 3} \varsigma_{1}(t-d(t)) ;
$$

then, from (46), a scalar $m>0$ can be found such that, for any $t>0$,

$$
\|e(t)\|^{2} \leq m e^{-\varepsilon t}\|\phi(t)\|_{d}^{2} .
$$

To study the exponential stability of $\varsigma_{2}(t)$, we construct a function as

$$
\begin{aligned}
J(t)= & \varsigma_{2}(t)^{T} Q_{22} \varsigma_{2}(t) \\
& -\varsigma_{2}(t-d(t))^{T} Q_{22} \varsigma_{2}(t-d(t)) .
\end{aligned}
$$

By premultiplying the second equation of (41) with $\varsigma_{2}(t)^{T} P_{4}^{T}$, we get that

$$
\begin{gathered}
0=2\left[\varsigma_{2}(t)^{T} P_{4}^{T} \varsigma_{2}(t)+\varsigma_{2}(t)^{T} P_{4}^{T} \alpha(t) A_{d 4} \varsigma_{2}(t-d(t))\right. \\
\left.+\varsigma_{2}(t)^{T} P_{4}^{T} e(t)\right] .
\end{gathered}
$$

Adding (50) to (49) yields that

$$
\begin{aligned}
J(t)= & \varsigma_{2}(t)^{T}\left(P_{4}^{T}+P_{4}+Q_{22}\right) \varsigma_{2}(t) \\
& +2 \varsigma_{2}(t)^{T} P_{4}^{T} \alpha(t) A_{d 4} \varsigma_{2}(t-d(t)) \\
& -\varsigma_{2}(t-d(t))^{T} Q_{22} \varsigma_{2}(t-d(t)) \\
& +2 \varsigma_{2}(t)^{T} P_{4}^{T} e(t) \\
\leq & {\left[\varsigma_{2}(t) \varsigma_{2}(t-d(t))\right]^{T} } \\
& \times\left[\begin{array}{cc}
P_{4}^{T}+P_{4}+Q_{22} & \alpha P_{4}^{T} A_{d 4} \\
* & -Q_{22}
\end{array}\right]\left[\begin{array}{c}
\varsigma_{2}(t) \\
\varsigma_{2}(t-d(t))
\end{array}\right] \\
& +\eta_{1} \varsigma_{2}(t)^{T} \varsigma_{2}(t)+\eta_{1}^{-1} e(t)^{T} P_{4} P_{4}^{T} e(t),
\end{aligned}
$$

where $\eta_{1}$ is any positive scalar.

On the other hand, we can get from (17b) that

$$
\left[\begin{array}{cc}
\mathrm{Q}-E^{T} Z E+P^{T} A+A^{T} P & \alpha P^{T} A_{d}+E^{T} Z E-E^{T} Y E \\
* & -\mathrm{Q}+E^{T} Y E+E^{T} Y^{T} E-2 E^{T} Z E
\end{array}\right]
$$$$
<0 \text {. }
$$

Premultiplying and postmultiplying (52) by $\left[\begin{array}{cc}H & 0 \\ 0 & H\end{array}\right]^{T}$ and $\left[\begin{array}{cc}H & 0 \\ 0 & H\end{array}\right]$, respectively, a scalar $\eta_{2}>0$ can be found such that

$$
\left[\begin{array}{cc}
P_{4}^{T}+P_{4}+Q_{22} & \alpha P_{4}^{T} A_{d 4} \\
* & -Q_{22}
\end{array}\right] \leq-\left[\begin{array}{cc}
\eta_{2} I & 0 \\
0 & 0
\end{array}\right] .
$$

On the other hand, since $\eta_{1}$ can be chosen arbitrarily, $\eta_{1}$ can be chosen small enough such that $\eta_{2}-\eta_{1}>0$. Then, a scalar $\eta_{3}>1$ can always be found such that

$$
Q_{22}-\left(\eta_{2}-\eta_{1}\right) I \geq \eta_{3} Q_{22}
$$

It follows from (48), (49), (51), (53), and (54) that

$$
\begin{aligned}
\varsigma_{2}(t)^{T} Q_{22} \varsigma_{2}(t) \leq & \eta_{3}^{-1} \varsigma_{2}(t-d(t))^{T} Q_{22} \varsigma_{2}(t-d(t)) \\
& +\left(\eta_{1} \eta_{3}\right)^{-1} e(t)^{T} P_{4} P_{4}^{T} e(t)
\end{aligned}
$$

which infers

$$
f(t) \leq \eta_{3}^{-1} \sup _{t-d \leq s \leq t} f(t)+\Xi e^{-\delta t}
$$

where $0<\delta<\min \left\{\varepsilon, d^{-1} \ln \eta_{3}\right\}, f(t)=\varsigma_{2}(t)^{T} Q_{22} \varsigma_{2}(t)$, and $\xi=\left(\eta_{1} \eta_{3}\right)^{-1} m\left\|P_{4}\right\|^{2}\|\phi(t)\|_{d}^{2}$.

Applying Lemma 7 to (56) yields that

$$
\begin{gathered}
\left\|\varsigma_{2}(t)\right\|^{2} \leq \lambda_{\min }^{-1}\left(Q_{22}\right) \lambda_{\max }\left(Q_{22}\right) e^{-\delta t}\left\|\varsigma_{2}(t)\right\|_{d}^{2} \\
+\frac{\lambda_{\min }^{-1}\left(Q_{22}\right) \xi e^{-\delta t}}{1-\eta_{3}^{-1} e^{\delta d}} .
\end{gathered}
$$

We can find from (46) and (57) that system (11) is exponentially stable. This completes the proof. 
Next, we will present the robust stability criterion for the following system:

$$
\begin{aligned}
E \dot{x}(t)= & (A+\Delta A) x(t) \\
& +\alpha(t)\left(A_{d}+\Delta A_{d}\right) x(t-d(t)) \\
x(t)= & \phi(t), \quad t \in[-\tau, 0],
\end{aligned}
$$

via Theorem 8 .

Theorem 9. Singular time-delay system (58) is robustly stable, if there exist a scalar $\varepsilon>0$, matrices $P, Y, Q>0, R>0$, and $Z>0$ such that

$$
\left[\begin{array}{ccccc}
\Xi_{11} & \Xi_{12} & E^{T} Y E & d A^{T} Z & P^{T} M \\
* & \Xi_{22} & -E^{T} Y E+E^{T} Z E & d \alpha A_{d}^{T} Z & 0 \\
* & * & -R & 0 & 0 \\
* & * & * & -Z & d M \\
* & * & * & * & -\varepsilon I
\end{array}\right]<0,
$$

where

$$
\begin{aligned}
& \Xi_{11}=P^{T} A+A^{T} P+Q+R-E^{T} Z E+\varepsilon N_{1}^{T} N_{1}, \\
& \Xi_{12}=\alpha P^{T} A_{d}+E^{T} Z E-E^{T} Y E+\varepsilon \alpha N_{1}^{T} N_{2},
\end{aligned}
$$

$$
\begin{aligned}
\Xi_{22}= & -(1-\mu) Q+E^{T} Y E+E^{T} Y^{T} E-2 E^{T} Z E \\
& +\varepsilon \alpha^{2} N_{2}^{T} N_{2} .
\end{aligned}
$$

Proof. Condition (17b) with $A$ and $A_{d}$ replaced by $A+$ $M F(t) N_{1}$ and $A_{d}+M F(t) N_{2}$, respectively, can be written as

$$
\Xi+H F(t) \Phi+\Phi^{T} F(t)^{T} H^{T}<0,
$$

where $\Xi$ follows the same definition as in Theorem 8 and

$$
\begin{aligned}
& H=\left[\begin{array}{llll}
M^{T} P & 0 & 0 & d M^{T}
\end{array}\right], \\
& \Phi=\left[\begin{array}{llll}
N_{1} & \alpha N_{2} & 0 & 0
\end{array}\right] .
\end{aligned}
$$

By Lemma 6, (61) holds if there exists a scalar $\varepsilon>0$ such that

$$
\Xi+\varepsilon H H^{T}+\varepsilon^{-1} \Phi^{T} \Phi<0
$$

By Schur complement, (63) is equivalent to (59). Hence, according to Theorem 8 and Definition 4, system (58) is robustly stable. This completes the proof.

Theorem 10. Singular system (13) is robustly stable, if there exist a scalar $\varepsilon>0$, matrices $L, \widehat{Y}, \widehat{Q}>0, \widehat{R}>0$, and $\widehat{Z}>0$, and matrices $V$ with appropriate dimensions such that

$$
L^{T} E^{T}=E L \geq 0,
$$

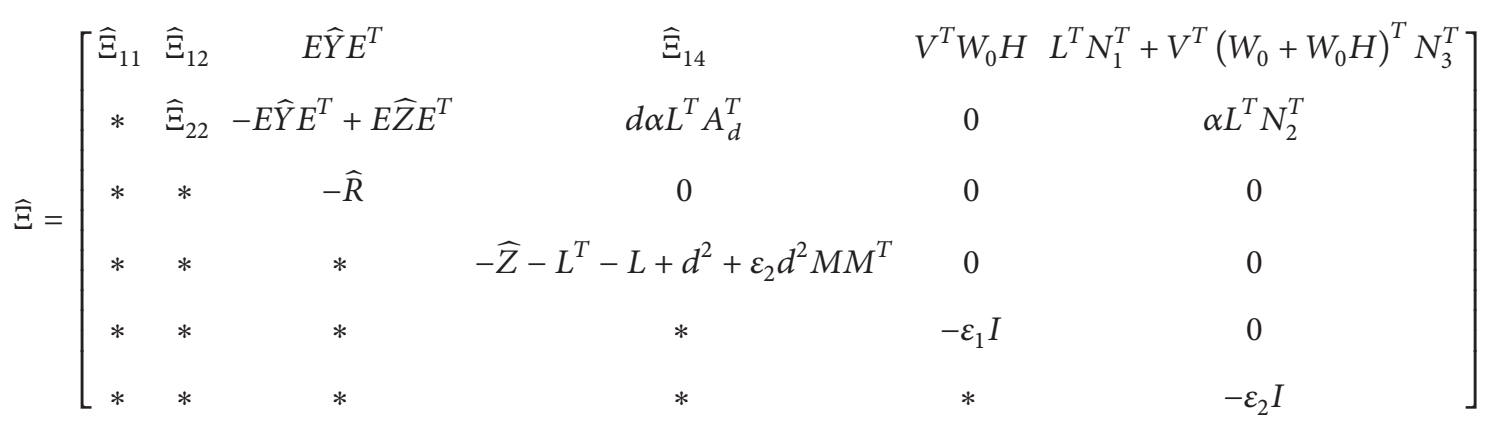

$$
\left[\begin{array}{cc}
\widehat{Z} & \widehat{Y} \\
* & \widehat{Z}
\end{array}\right]>0,
$$

where

$$
\begin{aligned}
\widehat{\Xi}_{11}= & A L+L^{T} A^{T}+B W_{0} V+V^{T} W_{0}^{T} B^{T}+\widehat{Q}+\widehat{R} \\
& -E \widehat{Z} E^{T}+\varepsilon_{1} B B^{T}+\varepsilon_{2} M^{T} M, \\
\widehat{\Xi}_{12}= & \alpha A_{d} L+E \widehat{Z} E^{T}-E \widehat{Y} E^{T},
\end{aligned}
$$

$$
\begin{aligned}
\widehat{\Xi}_{14}= & d\left(L^{T} A^{T}+V^{T} W_{0}^{T} B^{T}\right)+d B+\varepsilon_{2} d M M^{T}, \\
\widehat{\Xi}_{22}= & -(1-\mu) \widehat{Q}+E \widehat{Y} E^{T}+E \widehat{Y}^{T} E^{T} \\
& -2 E \widehat{Z} E^{T} .
\end{aligned}
$$

In this case, the desired controller gain is given as $K=$ $V L^{-1}$. 


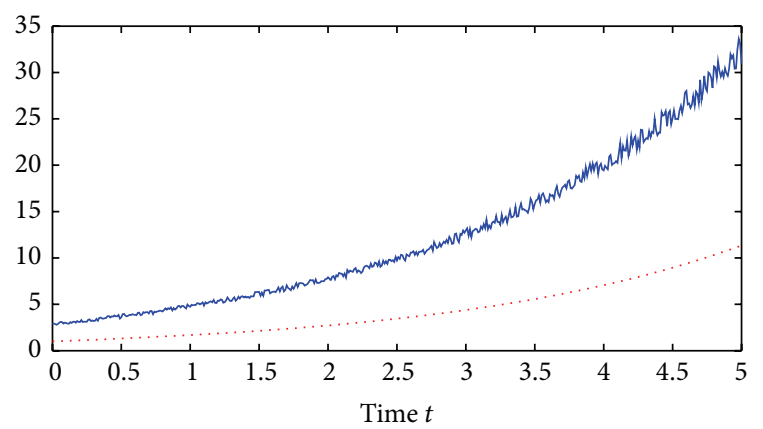

$x_{1}(t)$

$-x_{2}(t)$

FIGURE 1: State response of open-loop system.

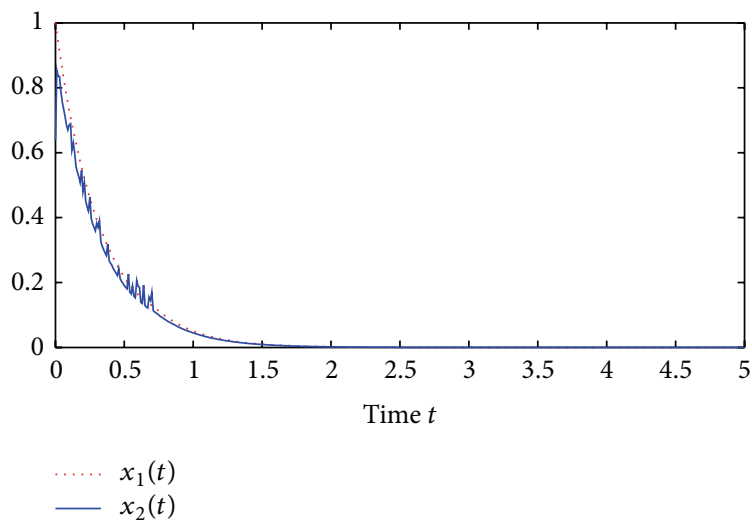

Figure 2: State response of closed-loop system.

Proof. By Condition (17b) with $A$ and $A_{d}$ replaced by $A+$ $B W K+M F(t) N_{1}+M F(t) N_{3} W K$ and $A_{d}+M F(t) N_{2}$, respectively, we have

$$
\begin{aligned}
& {\left[\begin{array}{cccc}
\bar{\Xi}_{11} & \bar{\Xi}_{12} & E^{T} Y E & d\left(A+B W K+M F(t) N_{1}+M F(t) N_{3} W K\right)^{T} Z \\
* & \Xi_{22} & -E^{T} Y E+E^{T} Z E & d \alpha\left(A_{d}+M F(t) N_{2}\right)^{T} Z \\
* & * & -R & 0 \\
* & * & * & -Z
\end{array}\right]} \\
& <0,
\end{aligned}
$$

where

$$
\begin{aligned}
\bar{\Xi}_{11} & \\
= & P^{T}\left(A+B W K+M F(t) N_{1}+M F(t) N_{3} W K\right) \\
& +\left(A+B W K+M F(t) N_{1}+M F(t) N_{3} W K\right)^{T} P \\
& +Q+R-E^{T} Z E, \\
& \quad \\
\bar{\Xi}_{12} &
\end{aligned}
$$$$
=\alpha P^{T}\left(A_{d}+M F(t) N_{2}\right)+E^{T} Z E-E^{T} Y E .
$$

Define matrix $J=\operatorname{diag}\left\{L, L, L, Z^{-1}\right\}, L=P^{-1}, \widehat{Q}=L^{T} Q L, \widehat{R}=$ $L^{T} R L, \widehat{Y}=L Y L^{T}, \widehat{Z}=L Z L^{T}$, and $V=K L$. Then, pre- and postmultiplying the left side of (63) by $J^{T}$ and $J$, respectively, we can find that

$$
\Sigma+H F(t) \Phi+\Phi^{T} F(t)^{T} H^{T}<0,
$$

where

$$
\begin{gathered}
\Sigma=\left[\begin{array}{cccc}
\overline{\bar{\Xi}}_{11} & \widehat{\Xi}_{12} & E \widehat{Y} E^{T} & d\left(L^{T} A^{T}+V^{T} W^{T} B^{T}\right) \\
* & \widehat{\Xi}_{22} & -E \widehat{Y} E^{T}+E \widehat{Z} E^{T} & d \alpha L^{T} A_{d}^{T} \\
* & * & -\widehat{R} & 0 \\
* & * & * & -L^{T} \widehat{Z}^{-1} L
\end{array}\right] \\
<0 \\
\overline{\bar{\Xi}}_{11}=A L+L^{T} A^{T}+B W V+V^{T} W^{T} B^{T}+\widehat{Q}+\widehat{R}-E \widehat{Z} E^{T} \\
H=\left[\begin{array}{llll}
L^{T} N_{1}^{T}+V^{T} W^{T} N_{3}^{T} & \alpha L^{T} N_{2}^{T} & 0 & 0
\end{array}\right]^{T} \\
\Phi=\left[\begin{array}{llll}
M^{T} & 0 & 0 & \left.d M^{T}\right] .
\end{array}\right.
\end{gathered}
$$

Noting $Z>0$, we have $-L^{T} \widehat{Z}^{-1} L \leq \widehat{Z}-L^{T}-L$. that

By Lemma 6, (68) holds if there exists a scalar $\varepsilon>0$ such

$$
\Psi=\Sigma+\varepsilon_{2} H H^{T}+\varepsilon_{2}^{-1} \Phi^{T} \Phi<0 .
$$

By Condition (70) with (10), we have

$$
\widehat{\Psi}=\left.\Psi\right|_{W=W_{0}(I+G)}
$$

$$
=\widehat{\Sigma}+\left[\begin{array}{c}
B \\
0 \\
0 \\
d
\end{array}\right]\left[W_{0} G V \quad 0 \quad 00\right]
$$

$$
+\left[\begin{array}{c}
V^{T} G^{T} W_{0}^{T} \\
0 \\
0 \\
0
\end{array}\right]\left[\begin{array}{llll}
B^{T} & 0 & 0 & d
\end{array}\right]+\varepsilon_{2} \widehat{H} \widehat{H}^{T}+\varepsilon_{2}^{-1} \Phi^{T} \Phi
$$$$
\leq \widetilde{\Sigma}+\varepsilon_{1}\left[\begin{array}{c}
B \\
0 \\
0 \\
d
\end{array}\right]\left[\begin{array}{l}
B \\
0 \\
0 \\
d
\end{array}\right]^{T}+\varepsilon_{1}^{-1}\left[\begin{array}{c}
V^{T} W_{0} H \\
0 \\
0 \\
0
\end{array}\right]\left[\begin{array}{c}
V^{T} W_{0} H \\
0 \\
0 \\
0
\end{array}\right]^{T}
$$

$$
+\varepsilon_{2} \widetilde{H} \widetilde{H}^{T}+\varepsilon_{2}^{-1} \Phi^{T} \Phi
$$

$$
=\check{\Sigma}+\varepsilon_{2} \widetilde{H} \widetilde{H}^{T}+\varepsilon_{2}^{-1} \widetilde{\Phi}^{T} \widetilde{\Phi}<0,
$$


where

$$
\begin{aligned}
& \widehat{\Sigma}=\left[\begin{array}{cccc}
\ddot{\Xi}_{11} & \widehat{\Xi}_{12} & E \widehat{Y} E^{T} & d\left(L^{T} A^{T}+V^{T}\left(W_{0}+W_{0} G\right)^{T} B^{T}\right) \\
* & \widehat{\Xi}_{22} & -E \widehat{Y} E^{T}+E \widehat{Z} E^{T} & d \alpha L^{T} A_{d}^{T} \\
* & * & -\widehat{R} & 0 \\
* & * & * & \widehat{Z}-L^{T}-L
\end{array}\right] \\
& \ddot{\Xi}_{11}=A L+L^{T} A^{T}+B\left(W_{0}+W_{0} G\right) V+V^{T}\left(W_{0}+W_{0} G\right)^{T} B^{T}+\widehat{Q}+\widehat{R}-E \widehat{Z} E^{T} \\
& \widetilde{\Sigma}=\left[\begin{array}{cccc}
\dot{\Xi}_{11} & \widehat{\Xi}_{12} & E \widehat{Y} E^{T} & d\left(L^{T} A^{T}+V^{T} W_{0}^{T} B^{T}\right) \\
* & \widehat{\Xi}_{22} & -E \widehat{Y} E^{T}+E \widehat{Z} E^{T} & d \alpha L^{T} A_{d}^{T} \\
* & * & -\widehat{R} & 0 \\
* & * & * & \widehat{Z}-L^{T}-L
\end{array}\right] \\
& \dot{\Xi}_{11}=A L+L^{T} A^{T}+B W_{0} V+V^{T} W_{0}^{T} B^{T}+\widehat{Q}+\widehat{R}-E \widehat{Z} E^{T} \\
& \check{\Sigma}=\left[\begin{array}{ccccc}
\widetilde{\bar{\Xi}}_{11} & \widehat{\Xi}_{12} & E \widehat{Y} E^{T} & d\left(L^{T} A^{T}+V^{T} W_{0}^{T} B^{T}\right)+d B & V^{T} W_{0} H \\
* & \widehat{\Xi}_{22} & -E \widehat{Y} E^{T}+E \widehat{Z} E^{T} & d \alpha L^{T} A_{d}^{T} & 0 \\
* & * & -\widehat{R} & 0 & 0 \\
* & * & * & \widehat{Z}-L^{T}-L+d^{2} & 0 \\
* & * & * & * & \varepsilon_{1} I
\end{array}\right] \\
& \widetilde{\bar{\Xi}}_{11}=A L+L^{T} A^{T}+B W_{0} V+V^{T} W_{0}^{T} B^{T}+\widehat{Q}+\widehat{R}-E \widehat{Z} E^{T}+\varepsilon_{1} B B^{T} \\
& \widehat{H}=\left[L^{T} N_{1}^{T}+V^{T}\left(W_{0}+W_{0} G\right)^{T} N_{3}^{T} \alpha L^{T} N_{2}^{T} \quad 0 \quad 0 \quad 0\right]^{T}
\end{aligned}
$$

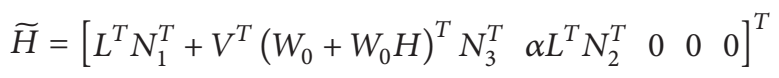

$$
\begin{aligned}
& \widetilde{\Phi}=\left[\begin{array}{lllll}
M^{T} & 0 & 0 & d M^{T} & 0
\end{array}\right] \text {. }
\end{aligned}
$$

By Schur complement, (71) is equivalent to (64a), (64b), and (64c). Hence, according to Theorem 8 and Definition 4, the system controlled by $u(t)=V L^{-1} x(t)$ is robustly stable. This completes the proof.

\section{Numerical Example}

In this section, we give an example to demonstrate the effectiveness of the proposed method. lows:

Consider the singular system (1) with parameters as fol-

$$
\begin{aligned}
E & =\left[\begin{array}{ll}
1 & 0 \\
0 & 0
\end{array}\right] \\
A & =\left[\begin{array}{ll}
1 & -0.2 \\
3 & -1.1
\end{array}\right] \\
A_{d} & =\left[\begin{array}{cc}
0.2 & 0 \\
0.1 & -0.1
\end{array}\right] \\
B & =\left[\begin{array}{ll}
1 & -0.1 \\
0 & 3
\end{array}\right] \\
M & =\left[\begin{array}{ll}
1 & 0 \\
0 & 1
\end{array}\right]
\end{aligned}
$$

$$
\begin{aligned}
& N_{1}=\left[\begin{array}{cc}
0.1 & 0 \\
0 & 0.1
\end{array}\right] \\
& N_{2}=\left[\begin{array}{cc}
0.1 & 0 \\
0 & 0.1
\end{array}\right] \\
& N_{3}=\left[\begin{array}{cc}
0.1 & 0 \\
0 & 0.1
\end{array}\right] .
\end{aligned}
$$

The state response of open-loop system $\left(u^{F}(t)=0\right)(1)$ is shown in Figure 1 and it is clear that the open-loop system is unstable.

In this example, we chose $\alpha=0.65, \mu=0.8$, and $d=0.8$. The first actuator failure matrix is $W=\operatorname{diag}\left\{w_{1}, w_{2}\right\}$, where $w_{1}^{\min }=0.7 \leq w_{1} \leq 1=w_{1}^{\max }$ and $w_{2}^{\min }=0 \leq w_{2} \leq$ $0.8=w_{2}^{\max }$ and the second actuator failure matrix is $W=$ $\operatorname{diag}\left\{w_{1}, w_{2}\right\}$, where $w_{1}^{\min }=0.1 \leq w_{1} \leq 0.6=w_{1}^{\max }$ and $w_{2}^{\min }=w_{2}=w_{2}^{\max }=0$.

For the first actuator failure, by Theorem 10, we have the following controller gain:

$$
K=\left[\begin{array}{cc}
-3.9595 & 0.009 \\
-0.2146 & -0.1379
\end{array}\right] .
$$

When actuator failures $\left(w_{1}=0.85\right.$ and $\left.w_{2}=0.4\right)$ with the designed feedback controller gain (74), the state response 


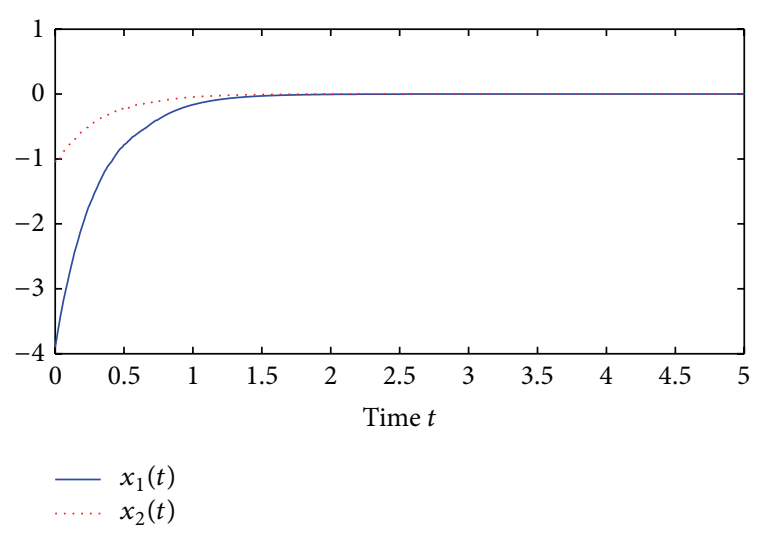

Figure 3: Controller output.

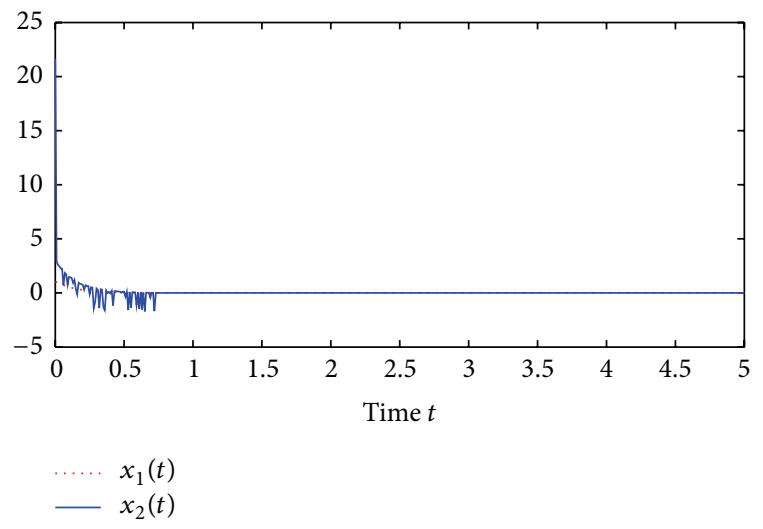

FIGURE 4: State response of closed-loop system.

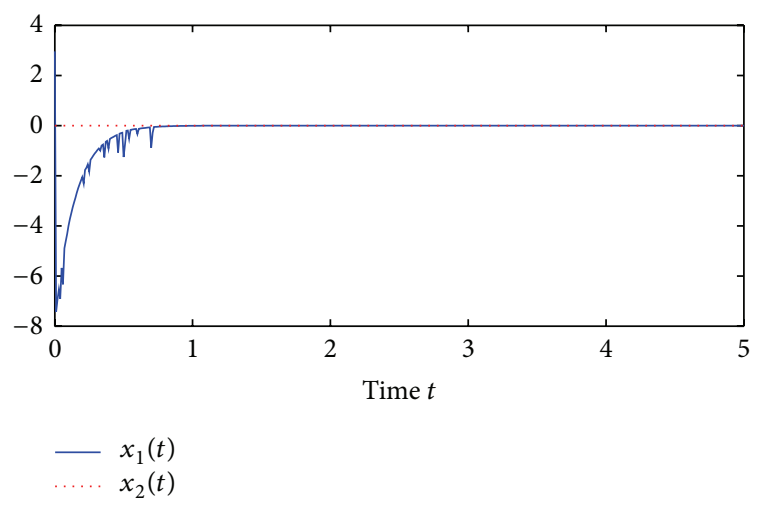

FIGURE 5: Controller output.

of the closed-loop system and the controller output are shown in Figures 2 and 3. In this example, all controllers are partially damaged. Figure 2 shows the reliable feedback control gain can guarantee that the state responses converge to zero asymptotically.

For the second actuator failure, by Theorem 10, we have the following controller gain:

$$
K=\left[\begin{array}{cc}
-12.3958 & 0.3135 \\
-0.3296 & -0.8185
\end{array}\right] .
$$

When actuator failures $\left(w_{1}=0.35\right.$ and $\left.w_{2}=1\right)$ with the designed feedback controller gain (75), the state response of the closed-loop system and the controller output are shown in Figures 4 and 5. In this example, not all controllers are partially damaged. Figure 4 shows the reliable feedback control gain can guarantee that the state responses converge to zero asymptotically.

\section{Conclusion}

The problems of reliable control for uncertain singular systems with randomly occurring time-varying delay and actuator faults have been studied. Based on the LMI approach, a sufficient condition has been established to ensure the considered systems are regular, impulse-free, and exponentially stable. The state feedback controller has been designed to ensure, for all possible actuator failures, the resultant closed-loop system is exponentially admissible. A numerical example has been provided to show the validness and less conservatism of the given result.

\section{Conflict of Interests}

The authors declare that there is no conflict of interests regarding the publication of this paper.

\section{References}

[1] Z. X. Li, H. Y. Su, Y. Gu, and Z. G. Wu, "Exponential stability analysis for discrete-time singular systems with randomly occurring delay," Circuits, Systems, and Signal Processing, vol. 32, no. 5, pp. 2231-2242, 2013.

[2] R. Lu, Y. Xu, and A. Xue, " $H_{\infty}$ filtering for singular systems with communication delays," Signal Processing, vol. 90, no. 4, pp. 1240-1248, 2010.

[3] S. Xu and J. Lam, "Robust stability and stabilization of discrete singular systems: an equivalent characterization," IEEE Transactions on Automatic Control, vol. 49, no. 4, pp. 568-574, 2004.

[4] J. H. Kim, "Delay-dependent robust $H_{\infty}$ filtering for uncertain discrete-time singular systems with interval time-varying delay," Automatica, vol. 46, no. 3, pp. 591-597, 2010.

[5] S. Zhu, Z. Cheng, and J. Feng, "Delay-dependent robust stability criterion and robust stabilization for uncertain singular timedelay systems," in Proceedings of the American Control Conference (ACC '05), pp. 2839-2844, June 2005.

[6] R. Lu, H. Li, and Y. Zhu, "Quantized $H_{\infty}$ filtering for singular time-varying delay systems with unreliable communication channel," Circuits, Systems, and Signal Processing, vol. 31, no. 2, pp. 521-538, 2012.

[7] S. Zhu, C. Zhang, Z. Cheng, and J. Feng, "Delay-dependent robust stability criteria for two classes of uncertain singular time-delay systems," IEEE Transactions on Automatic Control, vol. 52, no. 5, pp. 880-885, 2007.

[8] Y. He, Q.-G. Wang, C. Lin, and M. Wu, "Delay-range-dependent stability for systems with time-varying delay," Automatica, vol. 43, no. 2, pp. 371-376, 2007.

[9] X. Mao, N. Koroleva, and A. Rodkina, "Robust stability of uncertain stochastic differential delay equations," Systems \& Control Letters, vol. 35, no. 5, pp. 325-336, 1998. 
[10] Y. Wang, Z. Wang, and J. Liang, "Global synchronization for delayed complex networks with randomly occurring nonlinearities and multiple stochastic disturbances," Journal of Physics A: Mathematical and Theoretical, vol. 42, no. 13, Article ID 135101, 2009.

[11] D. Ding, Z. Wang, B. Shen, and H. Shu, " $H_{\infty}$ state estimation for discrete-time complex networks with randomly occurring sensor saturations and randomly varying sensor delays," IEEE Transactions on Neural Networks and Learning Systems, vol. 23, no. 5, pp. 725-736, 2012.

[12] R. Lu, H. Wu, and J. Bai, "New delay-dependent robust stability criteria for uncertain neutral systems with mixed delays," Journal of the Franklin Institute: Engineering and Applied Mathematics, vol. 351, no. 3, pp. 1386-1399, 2014.

[13] L. Wu and W. X. Zheng, "Passivity-based sliding mode control of uncertain singular time-delay systems," Automatica, vol. 45, no. 9, pp. 2120-2127, 2009.

[14] B. Shen, Z. Wang, H. Shu, and G. Wei, "On nonlinear $H_{\infty}$ filtering for discrete-time stochastic systems with missing measurements," IEEE Transactions on Automatic Control, vol. 53, no. 9, pp. 2170-2180, 2008. 


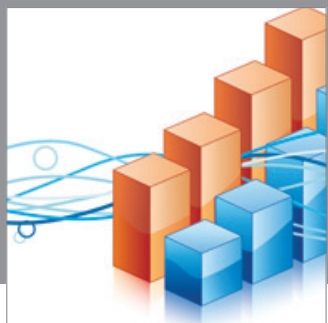

Advances in

Operations Research

mansans

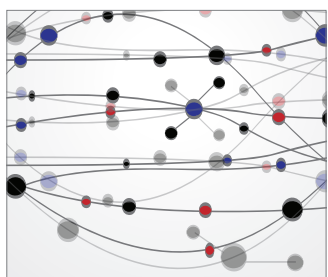

The Scientific World Journal
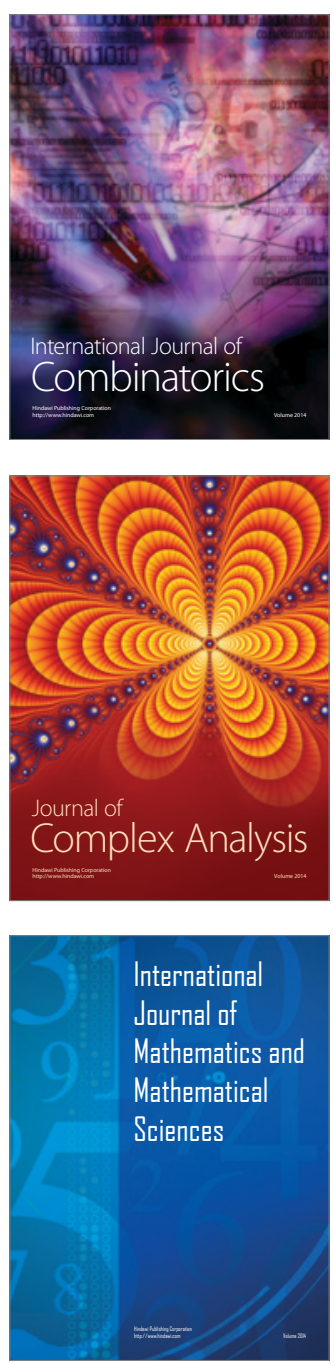
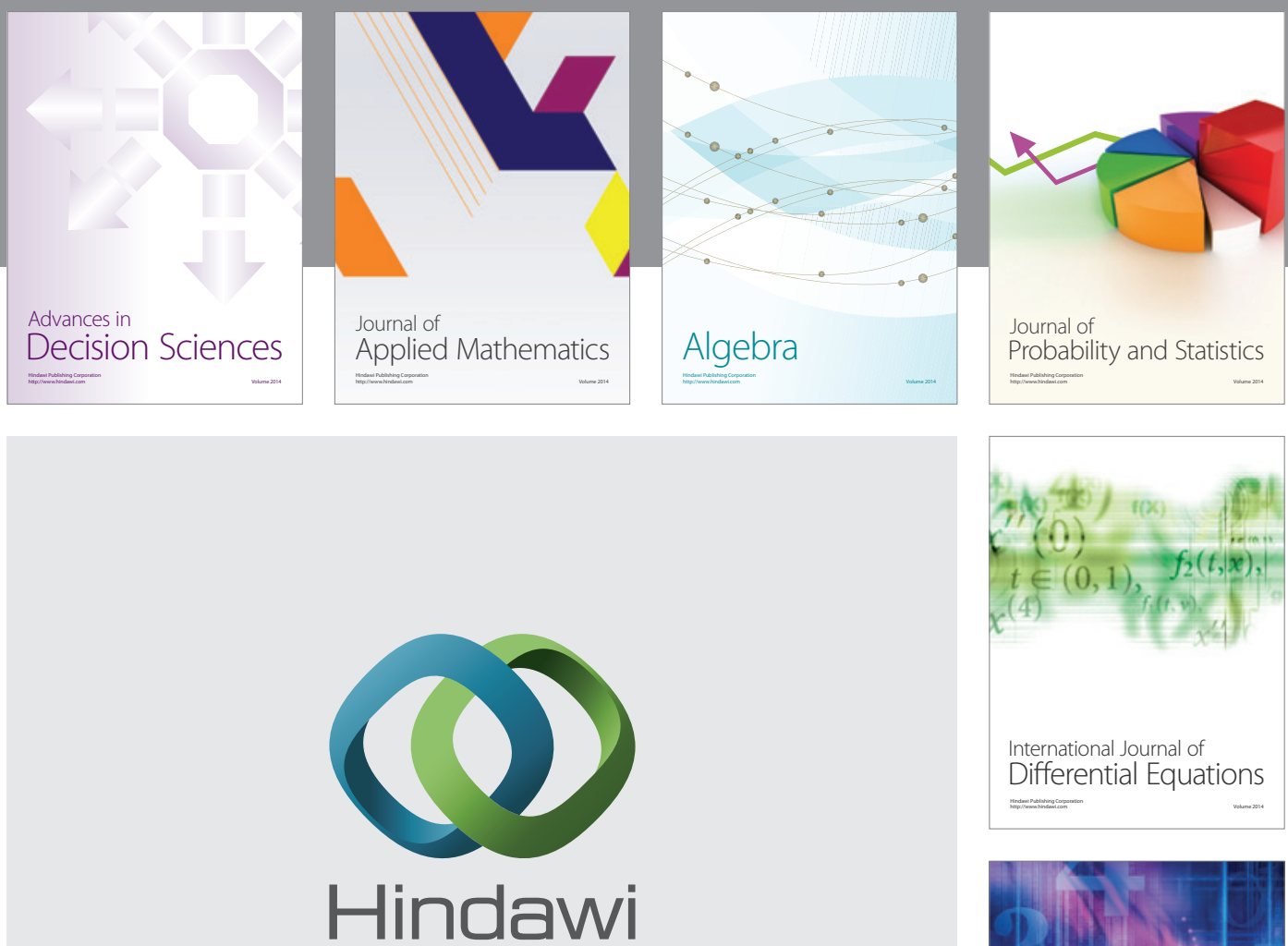

Submit your manuscripts at http://www.hindawi.com
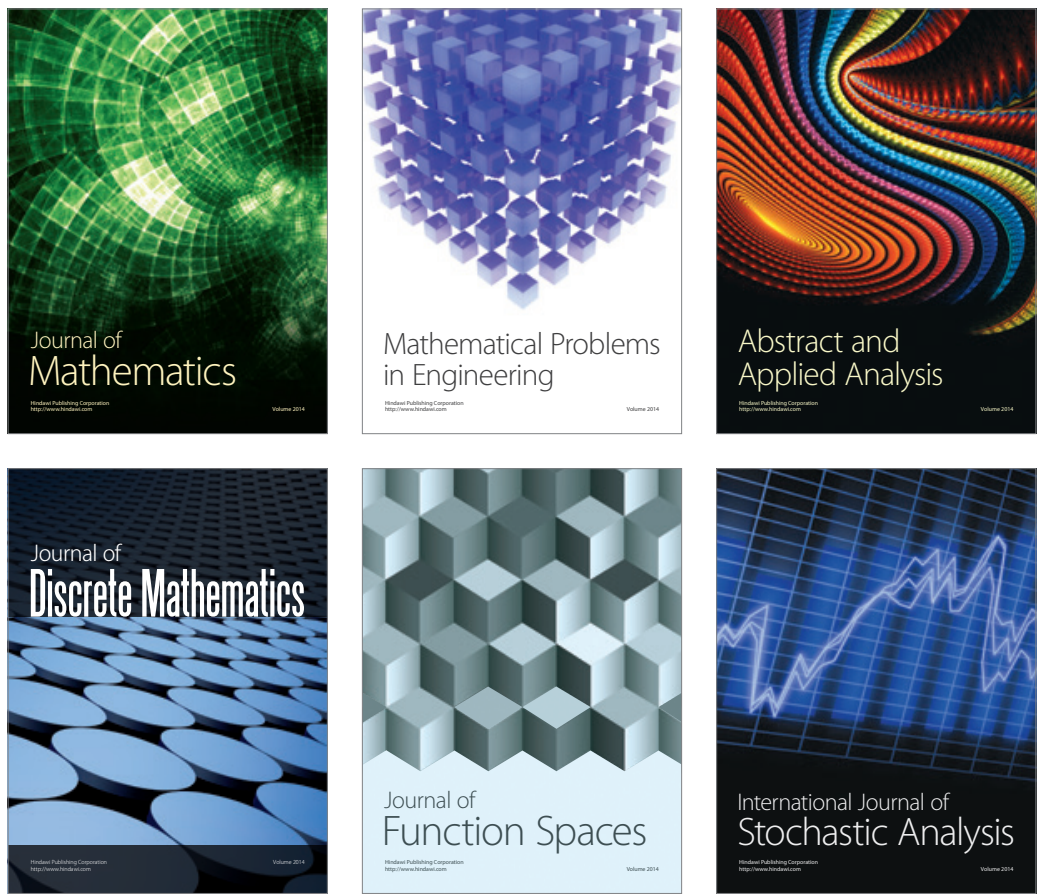

Journal of

Function Spaces

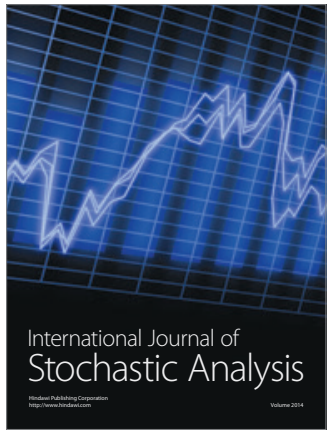

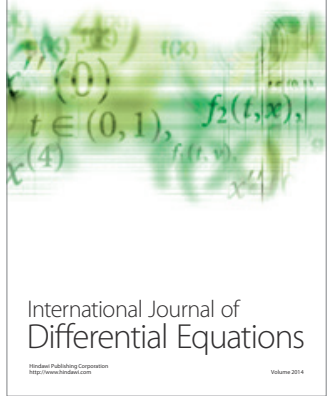
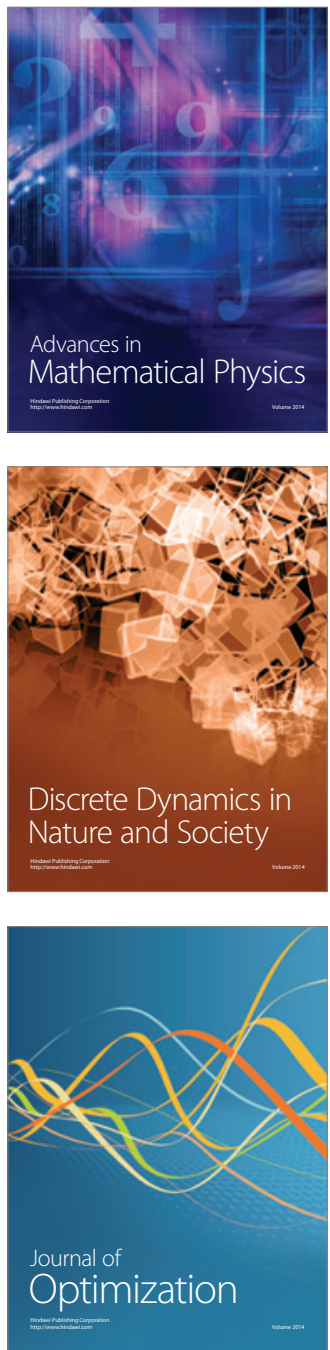